\title{
Isolation, characterization, and gene expression analysis of Wharton's jelly-derived mesenchymal stem cells under xeno-free culture conditions
}

This article was published in the following Dove Press journal:

Stem Cells and Cloning:Advances and Applications

20 April 20II

Number of times this article has been viewed

Parvathy Venugopal'

Sudha Balasubramanian'

Anish Sen Majumdar'

Malancha $\mathrm{Ta}^{2}$

'Stempeutics Research Pvt. Ltd, Manipal Hospital, Bangalore, India;

2Manipal Institute of Regenerative

Medicine, Manipal University,

Bangalore, India
Correspondence: Malancha Ta Manipal Institute of Regenerative Medicine, Manipal University, 10 Service

Road, Domlur Layout, Airport Road,

Bangalore-560 07I, India

Tel +9l 8025356663

Fax +9l 8025356662

Email malancha.ta@manipal.edu

\begin{abstract}
Mesenchymal stem cells (MSCs) have become an attractive tool for tissue engineering and targets in clinical transplantation due to their regeneration potential and immuno-suppressive capacity. Although MSCs derived from bone marrow are the most widely used, their harvest requires an invasive procedure. The umbilical cord, which is discarded at birth, can provide an inexhaustible source of stem cells for therapy. The Wharton's jelly-derived MSCs (WJ-MSCs), from the umbilical cord, have been shown to have faster proliferation rates and greater expansion capability compared with adult MSCs. The standard isolation and in vitro culture protocol for WJ-MSCs utilizes fetal bovine serum (FBS) or calf serum as a nutrient supplement. However, FBS raises potential safety concerns such as transmission of viral/prion disease and may initiate xenogeneic immune reactions against bovine antigens. Therefore, for therapeutic applications, there is an urgent requirement to establish an alternative nutrient supplement which would favor cell proliferation, retain MSC characteristics, and prove safe in human subjects. In the present study, we isolated and expanded WJ-MSCs in 5\% pooled, allogeneic human serum (HS) supplemented with $2 \mathrm{ng} / \mathrm{mL}$ of basic fibroblast growth factor. For cell dissociation, porcine trypsin was replaced with TrypLE, a recombinant enzyme, and a protease-free protocol was adapted for isolation of MSCs from WJ. We determined their growth kinetics, in vitro differentiation potential, surface marker expression, and colony-forming unit potential and compared them against standard WJ-MSC cultures expanded in 10\% FBS. All these parameters matched quite well between the two MSC populations. To test whether there is any alteration in gene expression on switching from FBS to HS, we analyzed a panel of stem cell and early lineage markers using Taqman ${ }^{\circledR}$ low density array. No significant deviation in gene expression was observed between the two populations. Thus we established an efficient, complete xeno-free protocol for propagation of human WJ-MSCs.
\end{abstract}

Keywords: human serum, explant, trypLE, umbilical cord, FBS, bFGF

\section{Introduction}

Mesenchymal stem cells (MSCs) are a rare population of multipotent precursors which can be isolated from many different tissue sources and also can differentiate to different lineages under appropriate induction conditions. MSCs represent an attractive cell source for therapeutic applications due to their potential for secreting bioactive molecules which are both trophic and immunomodulatory in nature. ${ }^{1}$ They were first described by Friedenstein and colleagues in 1976 in the stromal compartment of bone marrow. ${ }^{2,3}$ Human bone-marrow-derived MSCs (BM-MSCs), though extensively studied, involve a highly invasive procedure for harvesting, and the frequency, proliferation efficiency, and differentiation potential of BM-MSCs decline with age. ${ }^{4}$ 
As an alternative source of MSCs, Wharton's jelly (WJ) from umbilical cord possesses desirable characteristics such as a large, rapidly available donor pool, noninvasive and painless collection procedure, and ethically noncontroversial source of MSCs. WJ-MSCs have extensive in vitro expansion capabilities, wide multipotency, and do not induce teratomas. ${ }^{5}$ They are believed to be more primitive than MSCs derived from other tissue sources. ${ }^{6}$

Most of the existing protocols for in vitro culture of MSCs utilize fetal bovine serum (FBS) as a nutrient supplement and porcine trypsin for dissociating cells to generate single-cell suspensions. For safe therapeutic use of culture expanded stem cells in humans, use of animal-derived products in the culture is not preferred. MSCs cultured in FBS might pose a potential hazard which should not be overlooked. Bovine serum proteins and porcine macromolecules, if internalized in stem cells, are associated with the risk of transmitting viral/prion disease or can serve as antigenic substrates on transplanted cells and cause immunological reactions. ${ }^{7}$ In the past, severe anaphylaxis and immune reactions have been reported to be induced in the patients transplanted with the cells exposed to animal-derived products. ${ }^{8}$ It has been shown that a preparation of $10^{8}$ human MSCs grown under standard conditions in FBS would contain approximately $7-30 \mathrm{mg}$ of FBS proteins. ${ }^{9}$ Moreover, since FBS is a complex natural product and not well defined, lot-to-lot inconsistencies have been detected which could further lead to inconsistencies in MSC isolation, culture, and expansion. This makes it mandatory to prescreen individual lots of FBS for performance, which is not only cumbersome but also expensive. The use of autologous serum (AS) instead of FBS was recently shown to prevent life-threatening arrhythmias after cellular cardiomyoplasty. ${ }^{10}$ However, use of AS is practical only for protocols which involve low numbers of MSCs due to limited AS availability. MSCs are present at low frequency in their niche; hence, their expansion in vitro is essential for attaining a clinical dose. It is important to optimize MSC culture protocols which would result in maximal cell yields while preserving their basic characteristics. With respect to use of allogeneic, pooled human AB serum (HS) in MSC cultures, results have been contradictory. While a few studies reported growth arrest and death of MSCs after the first passage, ${ }^{8,11}$ a few others have been successful in isolating and expanding MSCs using AB serum. ${ }^{12-14}$ In fact, Le Blanc et al demonstrated that BM-MSC generated in $\mathrm{AB}$ serum suppressed lymphocyte proliferation in mixed lymphocyte cultures and after stimulation with phytohemagglutinin (PHA) as efficiently as those generated in $10 \%$ fetal calf serum (FCS). ${ }^{14}$ In this study, we have replaced
FBS with allogeneic, pooled HS for isolation and expansion of WJ-MSCs. Also, we reduced the pooled HS content while supplementing our cell culture medium with basic fibroblast growth factor (bFGF), which is a potent mitogen. Isolation of MSCs from umbilical cord matrix is usually achieved by collagenase, trypsin, and/or hyaluronidase enzyme digestion. Collagenase preparations produced in clostridium histolyticum are often characterized by batch-to-batch variability in terms of collagenolytic activity and endotoxin contamination. ${ }^{15}$ Recently, a few groups have reported successful isolation of MSCs from WJ without the use of any enzymatic treatment and with or without removal of the cord vessels. ${ }^{16-18}$ In this report, isolation of MSCs from the WJ of the umbilical cord, after removal of arteries and veins, has been carried out using a nonenzymatic procedure in which the use of proteases has been eliminated. Porcine trypsin has been replaced with TrypLE express, which is free of animal and human-derived components, for the dissociation of cells. Also, TrypLE does not require a neutralization step using serum-containing medium. Hence, for the first time, we report efficient, complete xeno-free propagation of human WJ-MSCs. Though still not serum-free, the current protocol makes our WJ-MSC product completely xeno-free.

We compared multiple parameters including growth kinetics, differentiation capabilities in vitro, immunosuppressive capacity and immunophenotype of WJ-MSCs propagated in allogeneic, pooled HS along with those cultured under standard FBS conditions. MSCs derived from various physiological tissue sources have their signature expression profile. To study whether there is any unusual shift in gene expression on substituting FBS with allogeneic HS, we performed a quantitative gene expression comparison using Taqman ${ }^{\circledR}$ low density array (TLDA) covering stem cell-specific and early lineage markers.

\section{Materials and methods Isolation and culture of WJ-MSC}

Fresh human umbilical cords $(\mathrm{n}=12)$ were obtained after full-term births (cesarean section or normal vaginal delivery) with informed consent using the guidelines approved by the Institutional Committee for Stem Cell Research and Therapy (ICSCRT) and Institutional Ethics Committee (IEC) at the Manipal Hospital, Bangalore, India. The umbilical cords were processed for isolation of WJ-MSCs as previously described, ${ }^{19}$ with some modifications. Briefly, after rinsing in normal saline $(0.9 \% \mathrm{w} / \mathrm{v}$ sodium chloride), the cords were aseptically stored at $4^{\circ} \mathrm{C}$ in sterile saline until processing. Next, the umbilical 
cord vessels were removed manually from cord segments, and the exposed mesenchymal tissue was cut into very small pieces or explants, approx. 1-2 mm, before placing them in a tissue culture dish. The explants were cultured in 1) DMEM-KO with $4500 \mathrm{mg} / \mathrm{mL}$ glucose and $2 \mathrm{mM}$ L-glutamine (Invitrogen, Carlsbad, CA), supplemented with 10\% FBS Cat.no.SH30084.03, Lot no.GRH0054 (Hyclone, Waltham, MA) and 2) DMEM-KO with $4500 \mathrm{mg} / \mathrm{mL}$ glucose and $2 \mathrm{mM}$ L-glutamine (Invitrogen), supplemented with $5 \%$ pooled, allogeneic HS and $2 \mathrm{ng} / \mathrm{mL}$ bFGF (SigmaAldrich, St Louis, MO). Pooled, allogeneic HS was collected from the blood bank of Manipal Hospital, Bangalore, India and filtered through a $0.2 \mu \mathrm{m}$ membrane before storing aliquots at $-20^{\circ} \mathrm{C}$. Once the cells reached $\sim 70 \%$ confluency, they were harvested and replated at 5000 cells $/ \mathrm{cm}^{2}$. The cells cultured in 10\% FBS were dissociated using $0.05 \%$ Trypsin-EDTA (Invitrogen), whereas cells cultured in HS were dissociated using TrypLE Express (Invitrogen).

\section{Proliferation kinetics}

The population doubling (PD) was determined using the formula:

$$
\mathrm{X}=[\log 10(\mathrm{NH})-\log 10(\mathrm{NI})] / \log 10(2),
$$

where NI is the inoculum cell number and NH the cell harvest number. PD for each passage was calculated and added to the $\mathrm{PD}$ of the previous passages in order to generate cumulative PD (CPD) data. The PD time was obtained by the formula:

$$
\mathrm{TD}=\operatorname{tp} \lg 2 /(\lg \mathrm{NH}-\lg \mathrm{NI}),
$$

where NI is the inoculum cell number, NH is the cell harvest number, and $\mathrm{t}$ is the time of the culture (in hours).

\section{Differentiation}

The multilineage differentiation potential of WJ-MSCs cultured in 5\% HS + $2 \mathrm{ng} / \mathrm{mL}$ bFGF versus $10 \% \mathrm{FBS}$ was determined by collecting cells at passage $3-5$ and subjecting them to induction medium favorable for osteogenic, chondrogenic, and adipogenic differentiation. For osteogenic differentiation, $60 \%-70 \%$ confluent cells were incubated in DMEM-KO medium supplemented with $10 \%$ (vol/vol) FBS (Hyclone) or $5 \%$ pooled, allogeneic HS, $2 \mathrm{mM} \mathrm{L-glutamine} \mathrm{(Invitrogen),}$ $0.1 \mu \mathrm{M}$ dexamethasone, $10 \mathrm{mM} \beta$ glycerophosphate, $0.2 \mathrm{mM}$ ascorbic acid (all reagents from Sigma-Aldrich). Mineralized deposits were visualized by Von Kossa staining after 21 days. Adipogenic differentiation was initiated in DMEM$\mathrm{KO} / 2 \mathrm{mM}$ glutamine supplemented with $200 \mu \mathrm{M}$ indomethacin, $0.5 \mathrm{mM} 3$-isobutyl-1-methylxanthine, $10 \mu \mathrm{g} / \mathrm{mL}$ insulin,
$1 \mu \mathrm{M}$ dexamethasone (all reagents from Sigma-Aldrich), and $10 \% \mathrm{FBS}$ or $5 \% \mathrm{HS}$. At day 18, the presence of lipid droplets was confirmed by oil red O staining (Sigma-Aldrich), and the nuclei were counterstained with hematoxylin.

Chondrogenic differentiation was induced in confluent monolayer cultures of WJ-MSCs using a STEMPRO chondrogenesis differentiation kit (Invitrogen). Differentiated cells were stained with Alcian blue 8GX (Sigma-Aldrich) after 18-20 days.

Images were captured using Nikon Eclipse TE2000-U inverted microscope (Nikon, Tokyo, Japan).

\section{Immunophenotyping}

Passage 2-4 WJ-MSCs cultured in 5\% HS $+2 \mathrm{ng} / \mathrm{mL}$ bFGF and 10\% FBS were stained with the following antihuman antibodies which mark the following cell surface markers: CD90-PE (0.2 mg/mL), CD73-PE (12.5 $\mu \mathrm{g} / \mathrm{mL})$, CD44-PE (12.5 $\mu \mathrm{g} / \mathrm{mL})$, CD34-PE $(12.5 \mu \mathrm{g} / \mathrm{mL})$, HLADRFITC $(25 \mu \mathrm{g} / \mathrm{mL})$, CD 40-PE-Cy7 $(24 \mu \mathrm{g} / \mathrm{mL})$, CD 80-PE (3.2 $\mu \mathrm{g} / \mathrm{mL}), \mathrm{CD} 86-\mathrm{PE}-\mathrm{Cy} 7(100 \mu \mathrm{g} / \mathrm{mL})$ (all from BD Pharmingen, Franklin Lakes, NJ), and CD105-PE $(50 \mu \mathrm{g} / \mathrm{mL})$ (R\&D systems, Minneapolis, MN). Cells incubated with identical concentrations of FITC-, PE-, or PE-Cy7-conjugated mouse IgG isotype antibodies (BD Pharmingen) served as negative controls. At least 10,000 events were acquired on BD FACSCalibur ${ }^{\circledR}$ flow cytometer, and the results were analyzed using WinMDI v2.8 software.

\section{Immunosuppression assay}

WJ-MSCs expanded in 5\% HS $+2 \mathrm{ng} / \mathrm{mL}$ bFGF or $10 \%$ FBS were treated with mitomycin $\mathrm{C}$ and plated in triplicates into 96-well plates at 12,500, 6250, 3125, and 1562 cells/ $\mathrm{mL}$. Peripheral blood mononuclear cells (PBMCs) were obtained from healthy donor's buffy coats by density gradient centrifugation, stimulated with $10 \mu \mathrm{g} / \mathrm{mL}$ phytohemagglutinin (PHA, Sigma-Aldrich) and added at $2 \times 10^{5}$ cells per well. After co-culture for 72 hours, cell proliferation was analyzed by the 3-(4,5-dimethylthiazol2-yl)-2,5-diphenyltetrazolium (MTT) (Sigma-Aldrich) assay.

The inhibition rate was calculated by the formula:

inhibition rate $(\%)=1-$ (average absorbance for treated group/average absorbance for control group) $\times 100$.

\section{CFU-F assay}

WJ-MSCs cultured in 5\% HS + $2 \mathrm{ng} / \mathrm{mL}$ bFGF and 10\% FBS, were seeded in duplicates, in 6-well tissue culture 
plates at a seeding density of 10 cells $/ \mathrm{cm}^{2}$. After 14 days, cells were washed with DPBS, fixed in $1 \%$ paraformaldehyde (Sigma-Aldrich) for 20 minutes, stained with $0.1 \%$ toluidine blue (in 1\% paraformaldehyde solution) for 1 hour, and then rinsed in tap water. Colonies consisting of a minimal cell number of 50 cells were counted. Data are reported as total colony number per 100 cells.

\section{Senescence assay}

Senescence assay was performed with passage 7-8 WJ-MSC cultures grown in $5 \% \mathrm{HS}+2 \mathrm{ng} / \mathrm{mL}$ bFGF and $10 \%$ FBS using Senescence $\beta$-Galactosidase Staining kit (Cell Signaling Technologies, Danvers, MA) according to the manufacturer's protocol. Cells were observed for development of blue color under a microscope using a10 $\times$ objective (Nikon). Ten random fields each from two different WJMSC cultures were used to calculate the percentage of positively stained cells. Representative microscopic fields were photographed.

\section{Reverse transcription-polymerase chain reaction (RT-PCR) analysis}

For the gene expression studies, WJ-MSCs from two different donors were cultured either in $5 \% \mathrm{HS}+2 \mathrm{ng} / \mathrm{mL}$ bFGF or $10 \%$ FBS. At passage $4-5$, total RNA was isolated from the WJ-MSCs using mirVana miRNA isolation kit (Ambion, Foster City, CA) as per manufacturer's instructions. It was next treated with DNA-free DNase I (Ambion) as per manufacturer's instructions, in order to eliminate traces of genomic DNA. Total RNA yield was estimated using Nanodrop 1000 (Thermo Scientific, Waltham, MA). RNA was then reverse-transcribed into cDNA using a high capacity cDNA reverse transcription kit (Applied Biosystems, Foster City, CA). PCR amplification was performed using Taq polymerase (Sigma-Aldrich) by following standard procedures. The primer sequences were as follows:

- COL 1a1 forward primer, 5'-AGGGCTCCA ACGAGATCGAGATCCG-3'; reverse primer, 5' - TACAGGAAGCAGACAGGGCCAACGTCG (222 bp)

- Desmin forward primer, 5'-CCAACAAGAACAAC GACG-3'; reverse primer, 5'-TGGTATGGACCTCA GAACC-3' (407 bp)

- LAMA-1 forward primer, 5'-CAGGACCCATTA CCCTTTTG-3'; reverse primer, 5'-GCCCTGCTTG GTTTCTTTATT-3' (139 bp)
- GABRB3 forward primer, 5'-CCTTGCCCAAAATCC CCTATGTCAAAGC-3'; reverse primer, 5'-GTATCG CCAATGCCGCCTGAGACCTC (276 bp)

- Runx2 forward primer, 5'-CCACCCGGCCGAA CTGGTCC-3'; reverse primer, 5'-CCTCGTCCGCTCC GGCCCACA-3' (257 bp)

- GATA-6 forward primer, 5'-GCCTCACTCCACTCGT GTTC-3'; reverse primer, 5'-TCAGATCAGCCACA CAATATGA-3' (546 bp)

- Nestin forward primer, 5'-AGAGGGGAATTCCT GGAG-3'; reverse primer, 5'-CTGAGGACCAGGACT CTCTA-3' (495 bp)

- GFAP forward primer, 5'-GGCCCGCCACTTGCAGGA GTACCAGG-3'; reverse primer, 5'-CTTCTGCTCG GGCCCCTCATGAGACG-3' (327 bp)

- Galanin forward primer, 5'-TGCGGCCCGAAGATGA CATGAAACC-3'; reverse primer, 5'-CCCAGGAGGCT CTCAGGACCGCTC-3' (187 bp)

- 18S forward primer, 5'-CGGCTACCACATCCAA GGAA-3'; reverse primer, 5'-GCTGGAATTACCG CGGCT-3' (186 bp)

\section{Taqman low density arrays}

The expression of a focused panel of pluripotent/stem cell and early lineage markers was analyzed using the Human Stem Cell Pluripotency Array (Applied Biosystems) which contains a well defined set of validated gene expression markers. cDNA was loaded on the microfluidic cards for thermal cycling on an ABI PRISM 7900HT Sequence Detection System (Applied Biosystems). Transcriptional analysis was performed on WJ-MSCs cultured in the two serum conditions, $5 \% \mathrm{HS}+2 \mathrm{ng} / \mathrm{mL}$ bFGF and $10 \% \mathrm{FBS}$. A cutoff cycle threshold $(\mathrm{Ct})$ value of 35.0 was arbitrarily assigned such that a $\mathrm{Ct}$ value above 35 was considered to be undetected.

The levels of gene expression of WJ-MSC grown in 5\% $\mathrm{HS}+\mathrm{bFGF}$ (target) relative to the level of expression in standard $10 \%$ FBS conditions (calibrator) was analyzed using the ABI PRISM 7900HT Sequence Detection System software (SDS v2.2 software) with the Comparative Ct Method $(\Delta \Delta \mathrm{Ct})$.

\section{Statistical methods}

Statistical tests and graphical representations were performed using GraphPad Prism 5 software (GraphPad, San Diego, $\mathrm{CA})$. Data are presented as mean \pm standard error mean, except for CFU-F and senescence data, which were expressed as mean \pm standard deviation. Statistical differences were calculated using Student's two-tailed $t$-test. $P<0.05$ was considered to be statistically significant. 


\section{Results}

\section{Isolation and expansion of WJ-MSCs under xeno-free conditions}

The explants culture protocol, as described above, allowed reproducible isolation of $\mathrm{WJ}-\mathrm{MSC}$ from umbilical cord $(n=12)$ without the use of any proteases to attain cellular dissociation. At the end of every passage, cells cultured in pooled, allogeneic HS were harvested using TrypLE Express, while trypsin was used for those cultured in $10 \%$ FBS. In an attempt to reduce the content of allogenic HS in the culture medium, we supplemented it with $2 \mathrm{ng} / \mathrm{mL}$ bFGF. This concentration of bFGF was shown to be the optimal condition for the culture of WJ-MSCs in our previous study. ${ }^{19}$ Ten percent FBS supplemented culture medium was used as reference. Therefore, WJ-MSCs were established in parallel cultures supplemented with either $5 \% \mathrm{HS}+2 \mathrm{ng} / \mathrm{mL}$ bFGF or $10 \%$ FBS. Cell morphology of WJ-MSCs cultured in FBS and pooled allogeneic $\mathrm{HS}+\mathrm{bFGF}$ was confirmed by phasecontrast microscopic examination as shown in Figures 1A and $1 \mathrm{~B}$, respectively. We estimated the total number of WJ-MSCs generated at the end of five passages. The cumulative cell yield ranged between $1.8 \times 10^{8} \pm 2.1 \times 10^{7}$ for $5 \%$ $\mathrm{HS}+2 \mathrm{ng} / \mathrm{mL}$ bFGF and $1.4 \times 10^{9} \pm 1.1 \times 10^{9}$ for $10 \% \mathrm{FBS}$ (Figure 1C). The difference was not significant $(P=0.39)$. The CPDs reached a peak of $10.4 \pm 0.4$ at passage 5 in $5 \%$ $\mathrm{HS}+2 \mathrm{ng} / \mathrm{mL}$ bFGF compared with $12.2 \pm 1.3(P>0.05)$ in $10 \%$ FBS (Figure 1D). Mean population doubling time of $35.3 \pm 2.4$ hours and $28.2 \pm 2.5$ hours $(P=0.113)$ was noted for $5 \% \mathrm{HS}+2 \mathrm{ng} / \mathrm{mL} \mathrm{bFGF}$ and $10 \% \mathrm{FBS}$, respectively (Figure 1E).

CFU-F assays were carried out to evaluate and compare the clonal expansion capacity of WJ-MSCs propagated in 5\% $\mathrm{HS}+2 \mathrm{ng} / \mathrm{mL}$ bFGF and $10 \% \mathrm{FBS}$, respectively (Figures $4 \mathrm{~L}$ and $4 \mathrm{M})$. WJ-MSCs in the two serum supplements demonstrated a similar colony forming potential with $21 \pm 0.7$ in $5 \% \mathrm{HS}+2 \mathrm{ng} / \mathrm{mL}$ bFGF and $18.7 \pm 3.8$ in standard medium containing $10 \% \mathrm{FBS}$.

\section{Surface phenotype characterization and comparison of xeno-free and standard FBS cultured WJ-MSCs}

To compare the immunophenotype profile of WJ-MSCs expanded in $5 \% \mathrm{HS}+2 \mathrm{ng} / \mathrm{mL}$ bFGF with those cultured in the standard medium, cell surface markers were analyzed by flow cytometry (Figures 2 and 3 ). All the surface markers tested were almost equally expressed on the cells irrespective of the serum supplement used in the composition of the culture medium. Both the populations of WJ-MSCs were positive for mesenchymal markers CD 44, CD 73, CD 90, and CD 105 and negative for CD 34, HLA-DR, and costimulatory molecules (CD 40, CD 80, and CD 86).

\section{Multilineage differentiation capacity of xeno-free WJ-MSCs}

WJ-MSCs expanded in 5\% HS + $2 \mathrm{ng} / \mathrm{mL}$ bFGF and under the standard condition of $10 \% \mathrm{FBS}$, as reference, were investigated for their in vitro differentiation capacity along adipogenic (Figures 4A-4C), osteogenic (Figures 4D-4F), and chondrogenic lineages (Figures 4G-4I). Cells at passage 3-5 were used. No detectable difference in staining was observed between the two populations.

\section{Immunosuppression by xeno-free WJ-MSCs}

To investigate whether WJ-MSCs cultured in the presence of $5 \% \mathrm{HS}+2 \mathrm{ng} / \mathrm{mL}$ bFGF are comparable to WJ-MSCs expanded in standard conditions of $10 \%$ FBS, in terms of their immunosuppression properties, we cocultured PHA-activated PBMCs with and without growth arrested WJMSCs and analyzed the effect of MSCs on proliferation of T cells in PBMCs. T cell proliferation was inhibited, and this was directly proportional to the number of WJ-MSCs in the coculture (Figure 5A). WJ-MSCs expanded in both 5\% HS + $2 \mathrm{ng} / \mathrm{mL}$ bFGF and $10 \%$ FBS were demonstrated to be immunosuppressive. At an MSC:PBMC ratio of 1:16, WJ-MSCs expanded in $5 \% \mathrm{HS}+2 \mathrm{ng} / \mathrm{mL}$ bFGF suppressed PBMC proliferation by $79 \% \pm 7.5 \%$, while those expanded in $10 \%$ FBS demonstrated $108 \% \pm 2.1 \%$ suppression $(P=0.052)$; at the other three dilutions, 1:32 $(P=0.13), 1: 64(P=0.87)$, and 1:128 $(P=0.87)$, similar degrees of suppression were achieved by both the WJ-MSC populations.

\section{Determination of senescence by $\beta$-galactosidase staining}

MSCs have a limited life span and when cultured in vitro for several passages, display replicative senescence, which is characterized by decreased proliferation, enlargement, replicative quiescence, and increase in SA- $\beta$-gal activity. We employed the enzyme lysosomal pH6 $\beta$-galactosidase as a senescence marker to get an estimate of the number of senescent WJ-MSCs cultured in 5\% HS $+2 \mathrm{ng} / \mathrm{mL}$ bFGF $(2.9 \% \pm 0.2 \%)$ versus that in $10 \% \operatorname{FBS}(2.6 \% \pm 1.2 \%)$ (Figures $4 \mathrm{~J}$ and $4 \mathrm{~K}$ ). The percentage of SA- $\beta$-gal positive cells as well as the intensity of staining did not differ significantly between the two populations. 

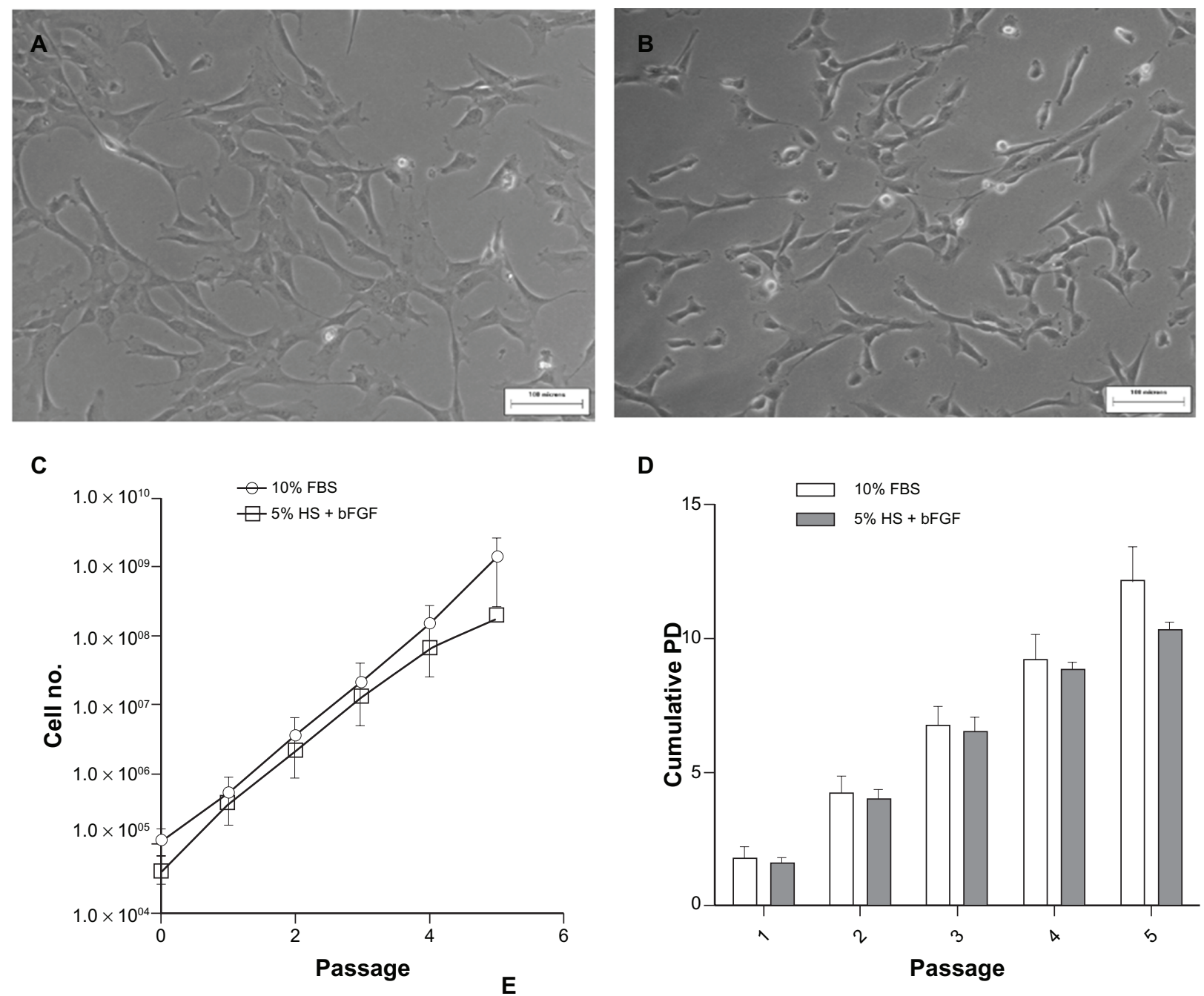

D
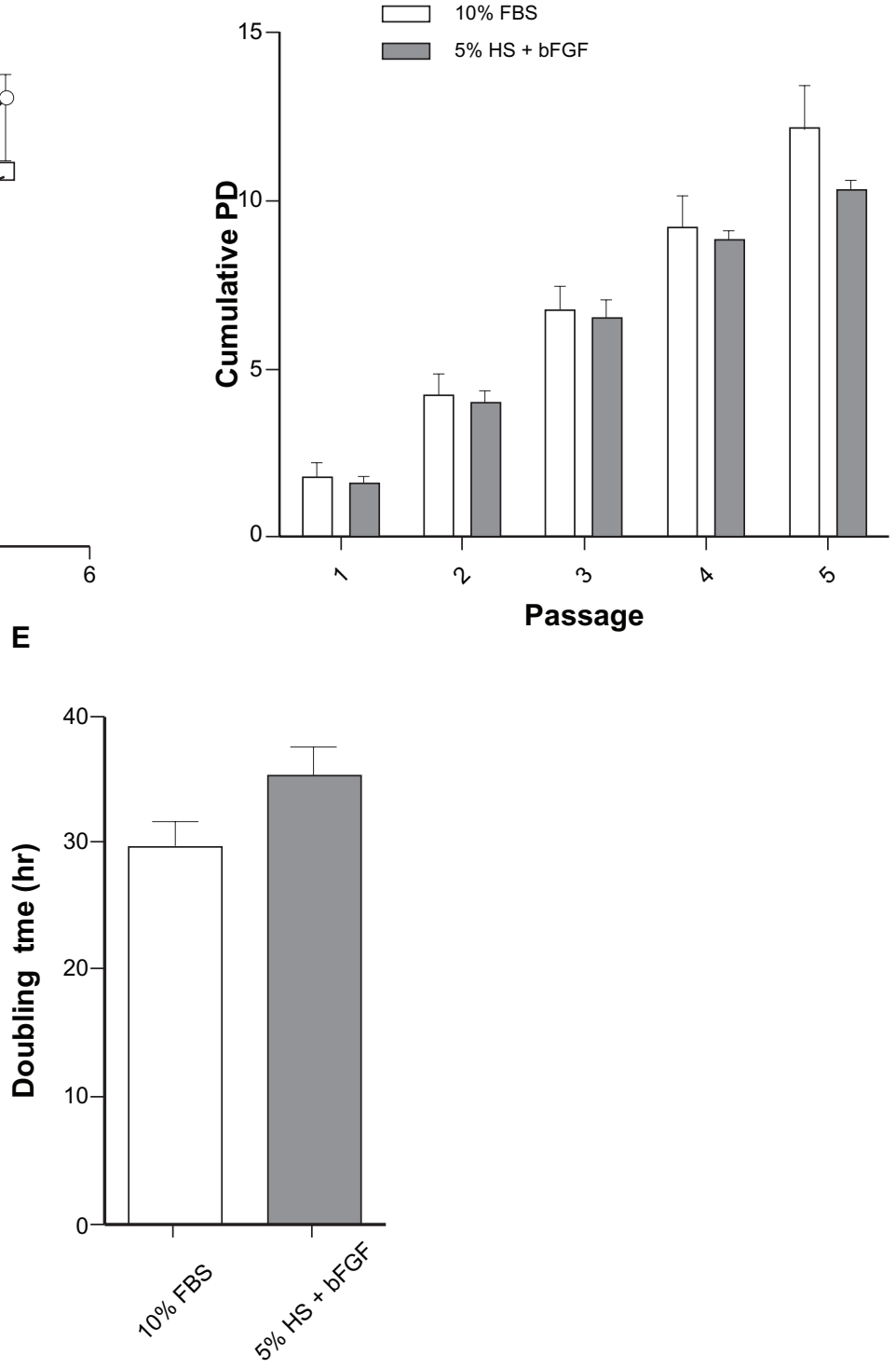

Figure I Morphology of passage $3 \mathrm{WJ}$-MSCs cultured in $5 \% \mathrm{HS}+2 \mathrm{ng} / \mathrm{mL}$ bFGF (A) and standard medium containing $10 \%$ FBS (B). Representative phase contrast images are presented. Comparison of total cell yield (C) and cumulative population doublings (D) over five passages in $5 \% \mathrm{HS}+2 \mathrm{ng} / \mathrm{mL}$ bFGF versus $10 \%$ FBS. Analysis of mean population doubling time (hour \pm SEM) of WJ-MSCs cultured in $5 \%$ HS $+2 \mathrm{ng} / \mathrm{mL}$ bFGF and standard medium containing $10 \%$ FBS (E). Results represent the average of three WJ-MSC samples $(n=3)$ with SEM. Scale bar $=100 \mu$ M.

Abbreviations: bFGF, basic fibroblast growth factor; FBS, fetal bovine serum; HS, human AB serum; SEM, standard error of the mean; WJ-MSC, Wharton's jelly-derived mesenchymal stem cell. 
$10 \%$ FBS
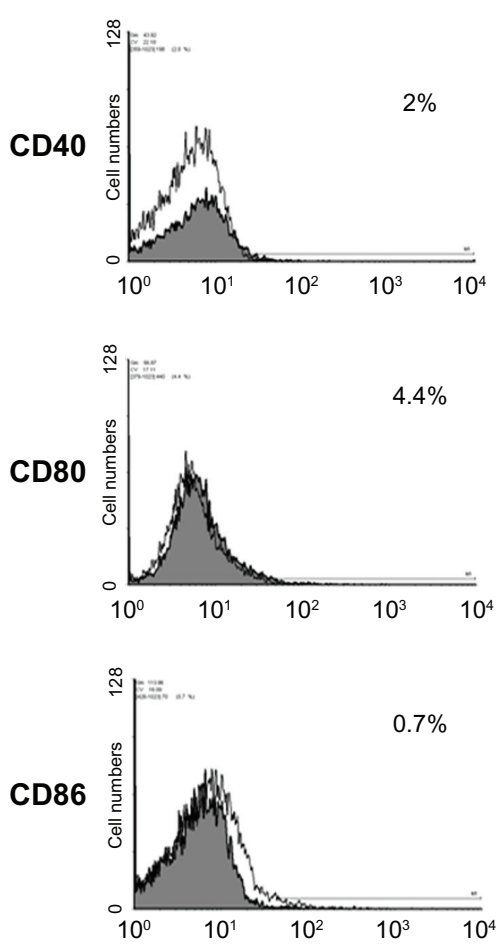

$5 \% \mathrm{HS}+\mathrm{bFGF}$
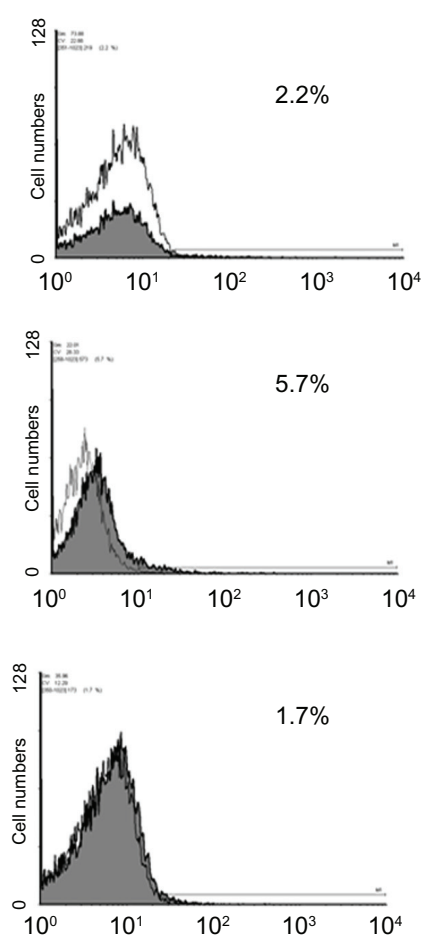

Raji
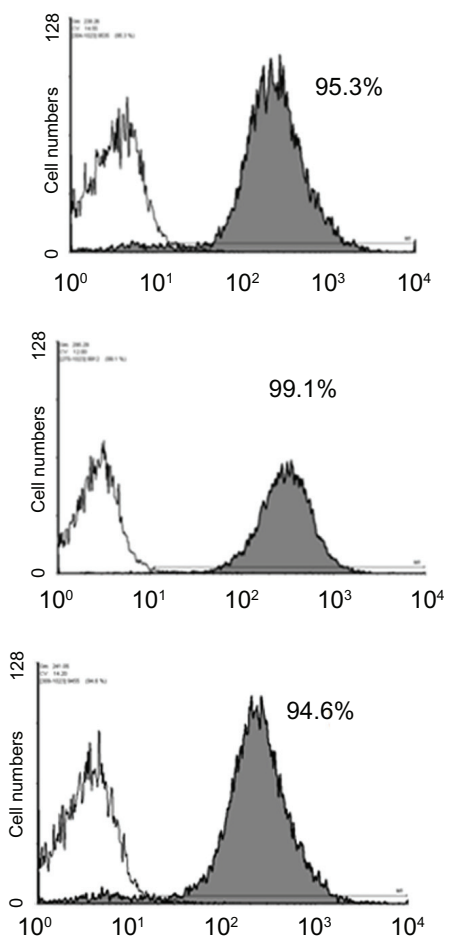

Figure 2 Detection of costimulatory molecules on WJ-MSCs by flow cytometry. Expression of costimulatory molecules (CD40, CD80, and CD86) on WJ-MSCs cultured in $5 \% \mathrm{HS}+2 \mathrm{ng} / \mathrm{mL}$ bFGF was analyzed by flow cytometry. Corresponding FBS cultures were analyzed for reasons of comparison. Raji cell line served as positive control for CD40, CD80, and CD86 staining. Representative histograms $(n=3)$ are demonstrated where shaded curves represent specific antibody signal.

Abbreviations: bFGF, basic fibroblast growth factor; FBS, fetal bovine serum; HS, human AB serum; WJ-MSC, Wharton's jelly-derived mesenchymal stem cell.

\section{Gene expression profiles of WJ-MSCs expanded in different serum preparations}

Stemness and early lineage specific-marker expression profile were analyzed for WJ-MSCs cultured in 5\% HS + $2 \mathrm{ng} / \mathrm{mL}$ bFGF and compared against reference WJ-MSCs expanded in $10 \%$ FBS. Although there was not much variation in the expression profile between WJ-MSCs cultured in $5 \%$ pooled, allogeneic $\mathrm{HS}+\mathrm{bFGF}$ and $10 \% \mathrm{FBS}$, some genes were differentially regulated between these two populations. Genes were considered significantly over-expressed in one population if their expression values were $\geq 1.5$-fold compared with the expression of the same gene in the other population. A list of the differentially regulated genes is presented in Table 1. The expression values are reported as the average of the values acquired from the two different donor samples cultured in 5\% HS $+2 \mathrm{ng} / \mathrm{mL}$ bFGF and $10 \%$ FBS, respectively. With respect to stem cell associated markers, WJ-MSCs cultured in 5\% HS $+2 \mathrm{ng} / \mathrm{mL}$ bFGF showed an upregulation for GAL, BRIX, FGF5, and PODXL and a downregulation for NANOG, LIN28, GABRB3, and CRABP2 compared with reference WJ-MSCs grown in $10 \% \mathrm{FBS}$. WJ-MSCs cultured in $5 \% \mathrm{HS}+2 \mathrm{ng} / \mathrm{mL}$ bFGF showed a higher expression for ectoderm markers GFAP,
NES, SEMA3A, and SYNAP and endothelial markers CD34 and PECAM while the endodermal genes, GATA6, LAMA, and SERPINA were downregulated compared with the $10 \%$ FBS cultured WJ cells.

\section{Validation of TLDA results with semi-quantitative RT-PCR}

In an attempt to validate our TLDA results, we selected seven random genes and obtained their gene expression levels for WJ-MSCs cultured in 5\% HS + $2 \mathrm{ng} / \mathrm{mL}$ bFGF versus $10 \%$ FBS by semi-quantitative RT-PCR (Figure 5B). Results were consistent with array data.

\section{Discussion}

The umbilical cord, which is discarded after birth, presents an attractive source of stem cells in regenerative medicine. The WJ within the umbilical cord is a rich source of MSCs and shares unique MSC properties such as wide multipotency, high proliferation rates, and hypoimmunogenicity. In contrast to BM-MSCs, they have higher proliferation rates, ${ }^{6}$ and their isolation does not involve invasive or painful procedures. Therefore, for serious evaluation of WJ as a potential source of MSCs and an alternative to the well characterized bone 
$5 \% \mathrm{HS}+\mathrm{bFGF}$
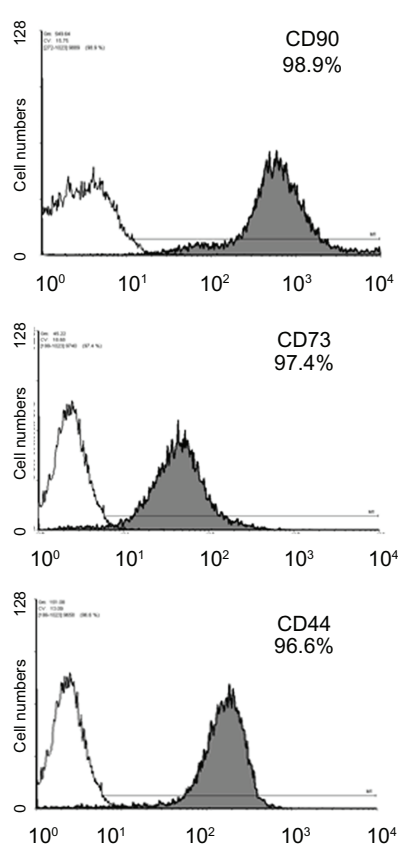

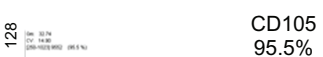
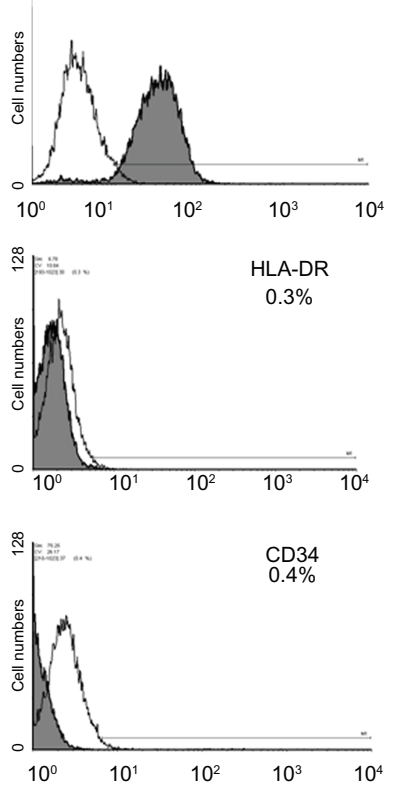

$10 \%$ FBS
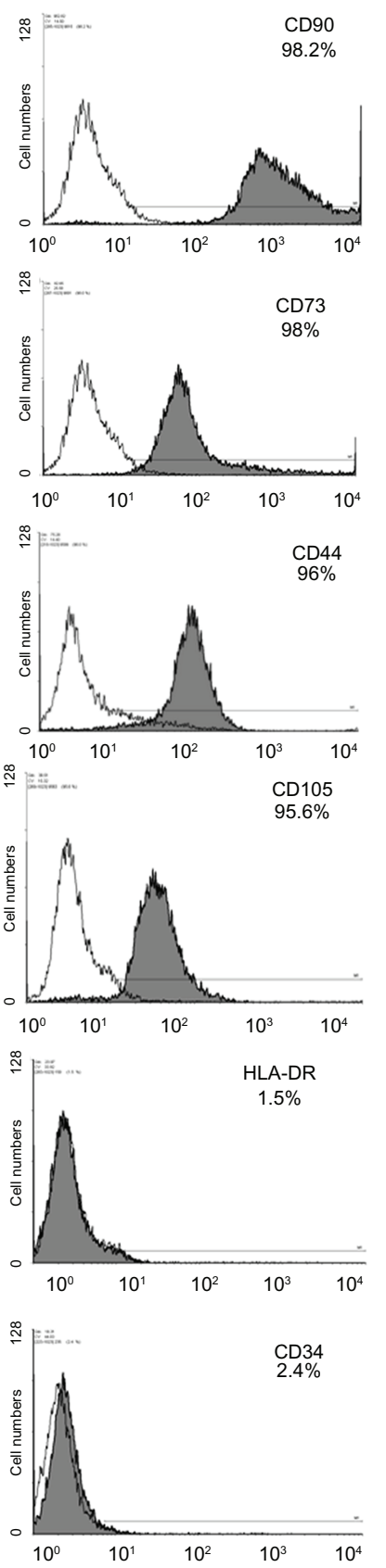

Figure 3 Flow cytometry analysis and comparison of the expression of surface markers by WJ-MSCs expanded using $5 \% \mathrm{HS}+2 \mathrm{ng} / \mathrm{mL}$ bFGF versus standard medium containing 10\% FBS. Representative histograms $(n=3)$ are demonstrated where shaded curves represent specific antibody signal.

Abbreviations: bFGF, basic fibroblast growth factor; FBS, fetal bovine serum; HS, human $A B$ serum; WJ-MSC, Wharton's jelly-derived mesenchymal stem cell.

marrow and cord blood-derived MSCs, a greater deal of attention has to be paid to turn them into a prospective source of stem cells for regenerative and cell-based transplantation therapies. The current most widely accepted culture protocols use FBS and porcine trypsin, which not only pose the risk of transmitting viral/prion disease, and unidentified zoonoses, but can also evoke severe immunological reactions in the recipient against xeno-proteins. Bovine serum albumin (BSA) and bovine apolipoprotein B-100, the key components (apoB100) of FBS, and porcine trypsin are potent immunogens, which can lead to potential adverse reactions in patients susceptible to bovine and porcine products. ${ }^{20}$ Hence, the use of animal-derived reagents for culture of MSCs for cellular therapy is undesirable. Therefore, for the potential use of WJ-MSCs for clinical applications in regenerative medicine, it is crucial to establish a protocol for the isolation and culture of WJ-MSCs that is completely free of xeno-compounds.

We earlier reported optimization of culture conditions for rapid clinical-scale expansion of WJ-MSCs and characterized the upscaled MSCs. ${ }^{19}$ Here we have explored the possibility of using pooled, allogeneic HS, at a reduced concentration rather than the standard condition of $10 \%$ FBS for in vitro isolation and expansion of WJ-MSCs. Propagation of WJ-MSCs in 5\% HS supplemented with $2 \mathrm{ng} / \mathrm{mL}$ bFGF was investigated in an effort to eliminate the potential risks associated with FBS, while a reduction from $10 \%$ to $5 \%$ was done to overcome the limited availability of HS and also to minimize the content of serum supplement in the cultures. Again, the use of proteases, such as collagenase, for digestion of the cord tissue was substituted by the explant method of isolation, which is based on the migratory ability of the MSCs from the cord matrix to the plastic surface of the tissue culture dish. Instead of porcine trypsin, a cell dissociation enzyme, TrypLE, which is animal origin free, was used for dissociation of cells. Earlier reports on the impact of using allogeneic HS on BM-MSCs are contradictory, since some studies demonstrated that allogeneic HS can support proliferation of MSCs while others reported a negative impact on cell proliferation, leading to MSC growth arrest and death. ${ }^{11,14,21}$ In the recent past, the isolation and culture of MSCs in serum and xeno-free media has been reported, though their formulations have not been disclosed. $22-24$

Here we have compared proliferation kinetics, surface antigen phenotype, in vitro differentiation potential, and gene expression of WJ-MSCs cultured using our optimized composition of $5 \% \mathrm{HS}+2 \mathrm{ng} / \mathrm{mL}$ bFGF against standard medium containing $10 \%$ FBS. Although FBS yielded higher cumulative cell numbers than $5 \% \mathrm{HS}+2 \mathrm{ng} / \mathrm{mL}$ bFGF at the 5 th passage, the difference was not significant. In our previous study, we optimized a protocol for scale-up to $10^{8} \mathrm{WJ}-\mathrm{MSC}$ from a single umbilical cord within a time period of 15 days. ${ }^{19}$ Therefore, there might not be a requirement for propagating the WJ-MSCs to late passages to achieve higher cell yield. In fact, an earlier report showed 

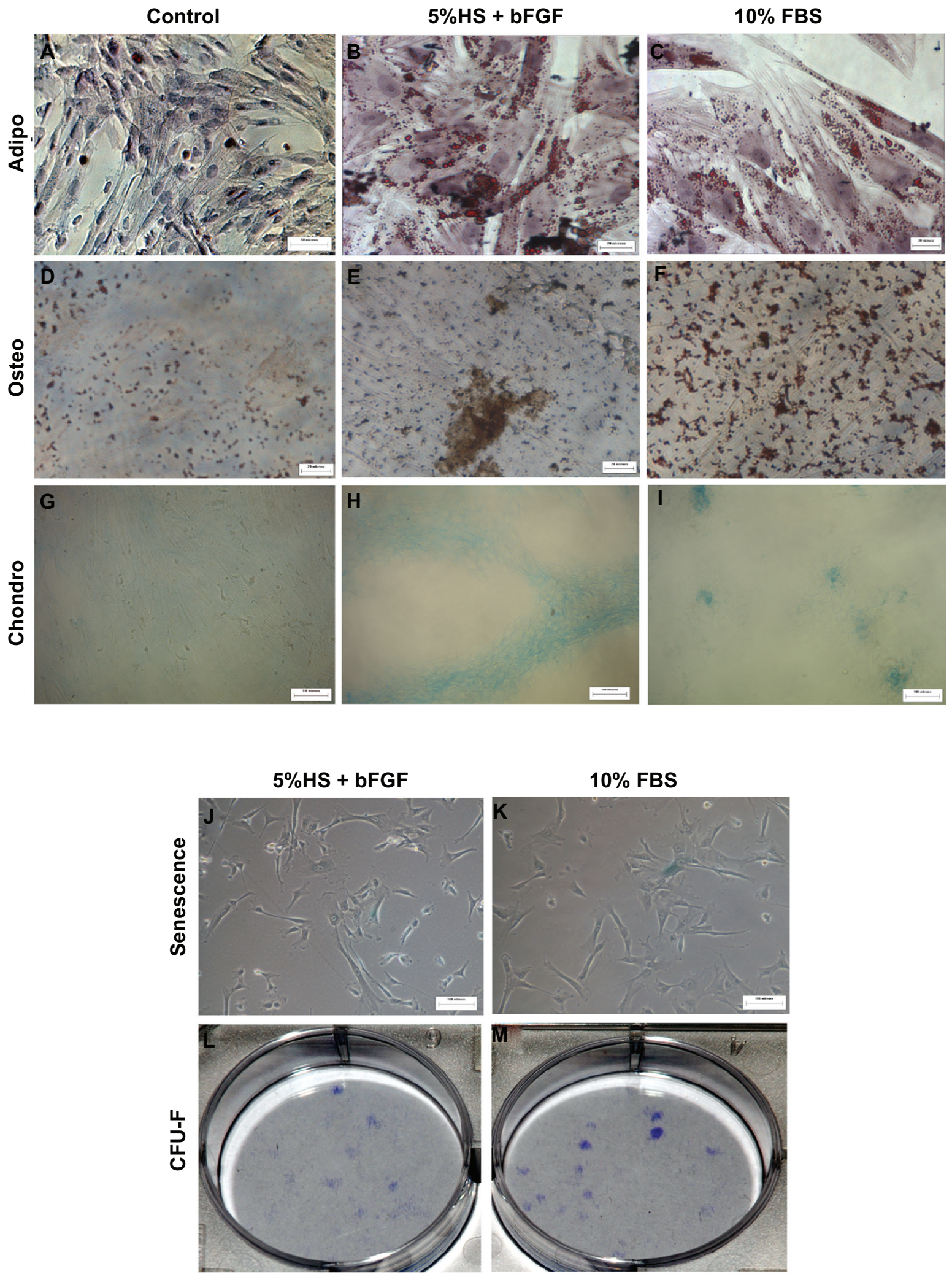

Figure 4 WJ-MSCs expanded in $5 \% \mathrm{HS}+2 \mathrm{ng} / \mathrm{mL}$ bFGF were subjected to adipogenic $(\mathrm{n}=3)(\mathbf{B})$, osteogenic $(n=2)(\mathbf{E})$, and chondrogenic differentiation $(\mathrm{n}=3)(\mathbf{H})$ and stained with Oil red O, Von Kossa, and alcian blue, respectively. Corresponding FBS cultures were induced to differentiate for reasons of comparison (C, F, and I). Noninduced control cultures without differentiation stimuli are shown in $\mathbf{A}, \mathbf{D}$, and $\mathbf{G}$. Senescence-associated $\beta$-galactosidase staining of WJ-MSCs $(n=2)$ at passage $7-8$, cultured in $5 \%$ HS $+2 \mathrm{ng} / \mathrm{mL}$ bFGF and $10 \% \mathrm{FBS}$, is demonstrated (J and $\mathbf{K}$ ). CFU-F colony formation (day 14) initiated from passage 3-4 WJ-MSCs expanded in $5 \% \mathrm{HS}+2 \mathrm{ng} / \mathrm{mL}$ bFGF and $10 \%$ FBS ( $\mathbf{L}$ and $\mathbf{M}$ ). Representative results of two independent WJ-MSC preparations are demonstrated. (Scale bar for A-F = $20 \mu M$; G-L $=100 \mu \mathrm{M}$.)

Abbreviations: bFGF, basic fibroblast growth factor; CFU-F, fibroblast colony forming units; FBS, fetal bovine serum; HS, human AB serum; WJ-MSC, Wharton's jelly-derived mesenchymal stem cell. 
A

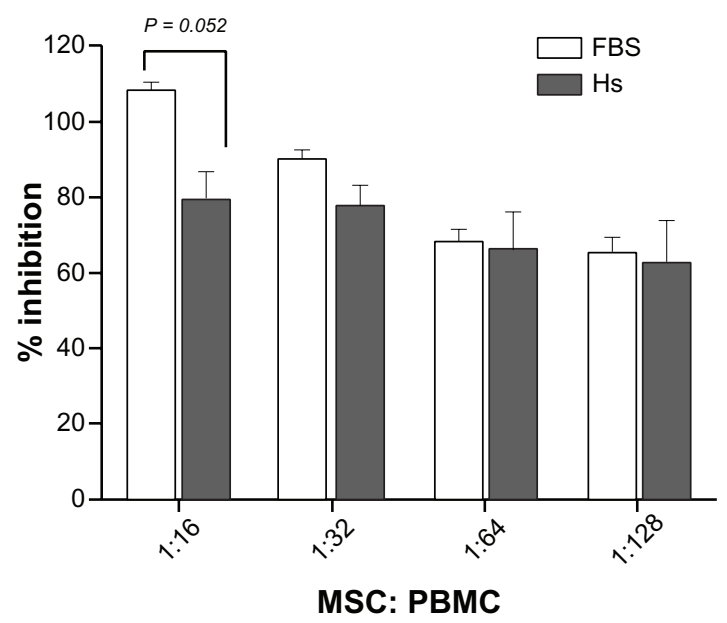

B

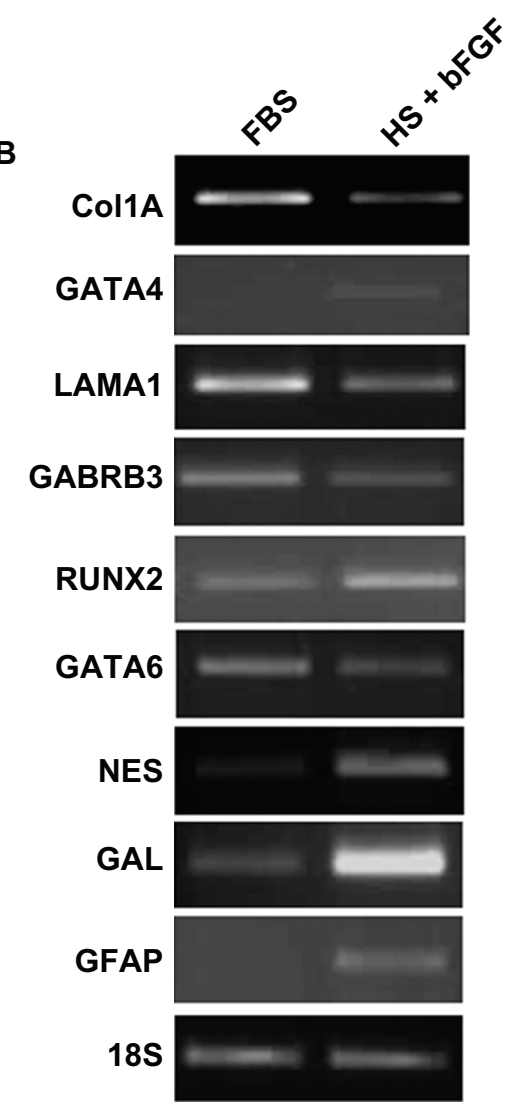

Figure 5 WJ-MSCs, expanded in $5 \%$ HS +2 ng/mL bFGF or 10\% FBS, were added in 1:16, I:32, I:64, and I:128 ratios to test their inhibitory effect on mitogen-activated PBMC proliferation, as demonstrated by MTT assay. MTT assays were performed in triplicate and the results were averaged. Results are representative of three independent experiments (A). The expression profile for a few selected genes from the TLDA results was validated by semi-quantitative RT-PCR. I8s rRNA was used as an internal control. Representative results of WJ-MSC preparation from two different donors are presented (B).

Abbreviations: bFGF, basic fibroblast growth factor; FBS, fetal bovine serum; HS, human AB serum; MSC, mesenchymal stem cell; MTT, 3-(4,5-dimethylthiazol-2-yl)-2,5diphenyltetrazolium; PBMC, peripheral blood mononuclear cell; RT-PCR, reverse transcription-polymerase chain reaction; TLDA, Taqman ${ }^{\circledR}$ low density array; WJ-MSC, Wharton's jelly-derived mesenchymal stem cell.

that MSCs enter senescence and start losing their stem cell characteristics almost undetectably from the moment in vitro cell culture begins. ${ }^{25}$ Therefore, ideally, MSCs should be considered at an early passage of in vitro culture for clinical applications.

A previous study on BM-MSCs reported an increase in proliferation rate along with downregulation of CD 44 and upregulation of HLA-class I and HLA-DR on addition of bFGF to the culture medium. ${ }^{26}$ However, in the case of WJ-MSCs, we did not notice this effect of bFGF on the expression levels of CD44 or HLA-DR in our previous study. ${ }^{19}$ Similarly, our present culture medium containing $5 \%$ HS supplemented with $2 \mathrm{ng} / \mathrm{mL}$ of bFGF did not alter the expression levels of either.

Human MSCs are negative for MHC class II and costimulatory molecules CD40, CD80, and CD86, which could render the $\mathrm{T}$ cells anergic and explain the immune privileged status of MSCs. ${ }^{27} \mathrm{WJ}-\mathrm{MSC}$ cultured in $5 \% \mathrm{HS}+2 \mathrm{ng} / \mathrm{mL}$ bFGF expressed the characteristic MSC surface markers and were negative for MHC class II (HLA-DR) and costimulatory molecules as determined by flow cytometry, and this profile was comparable to WJ-MSCs expanded in 10\% FBS. Next, we confirmed the inhibitory effect of WJ-MSCs on mitogenstimulated T-cell proliferation, and they displayed similar immunosuppressive capacity irrespective of the serum, HS or FBS, used for their culture. These data are in accordance with those reported earlier for BM-MSCs by Le Blanc and coworkers. $^{14}$

The WJ-MSCs maintained their characteristic morphology and clonal expansion capacity and retained the in vitro differentiation potential to adipogenic, osteogenic, and chondrogenic lineages when propagated in $5 \% \mathrm{HS}+2 \mathrm{ng} / \mathrm{mL}$ bFGF. WJ-MSCs grown in 10\% FBS supplemented medium was used as reference. Hence, WJ-MSCs expanded in $5 \%$ HS supplemented with $2 \mathrm{ng} / \mathrm{mL}$ bFGF met all criteria for MSCs.

In a previous study, we found an upregulation of undifferentiated stem cell markers and some early endodermal 
Table I Analysis of genes, differentially expressed by WJ-MSCs cultured in 5\% HS $2 \mathrm{ng} / \mathrm{mL}$ bFGF and standard medium containing I0\% FBS, using Taqman ${ }^{\circledR}$ low density array

\begin{tabular}{|c|c|c|c|c|}
\hline SI no. & Gene symbol & Gene name & Gene classification & $\begin{array}{l}\text { Fold change } \\
(\text { FBS }=I)\end{array}$ \\
\hline I & BRIX & Brix domain containing 2 & Stem cell marker & 1.54 \\
\hline 2 & CD34 & CD34 molecule & Endothelial & 2.02 \\
\hline 3 & COLIA & Collagen, type I, alpha I & Mesoderm & 0.41 \\
\hline 4 & CRABP2 & Retinoic acid-binding protein, cellular, type II & Stem cell marker & 0.51 \\
\hline 5 & FGF5 & Fibroblast growth factor 5 & Stem cell marker & 2.21 \\
\hline \multirow[t]{2}{*}{6} & GABRB3 & Gamma-aminobutyric acid (GABA) & Stem cell marker & 0.42 \\
\hline & & A receptor, beta 3 & & \\
\hline 7 & GAL & Galanin & Stem cell marker & 4.90 \\
\hline 8 & GATA4 & GATA binding protein 4 & Endoderm & 16.5 \\
\hline 9 & GATA6 & GATA binding protein 6 & Endoderm & 0.45 \\
\hline 10 & GFAP & Glial fibrillary acidic protein & Ectoderm/astrocyte & 3.2 \\
\hline 11 & LAMAI & Laminin, alpha I & Endoderm & 0.57 \\
\hline 12 & LIN28 & lin-28 homolog (C. elegans) & Stem cell marker & 0.33 \\
\hline 13 & NANOG & Nanog homeobox & Stem cell marker & 0.47 \\
\hline 14 & NES & Nestin & Ectoderm/neural & 1.64 \\
\hline 15 & NOG & Noggin & Mesoderm & 17.29 \\
\hline 16 & PECAMI & $\begin{array}{l}\text { Platelet/endothelial cell adhesion } \\
\text { molecule (CD3I antigen) }\end{array}$ & Endothelial & 4.0 \\
\hline 17 & PODXL & Podocalyxin-like & Stem cell marker & 1.94 \\
\hline 18 & PTEN & $\begin{array}{l}\text { Phosphatase and tensin homolog } \\
\text { (mutated in multiple advanced cancers I) }\end{array}$ & Stem cell marker & 1.94 \\
\hline 19 & RUNX2 & Runt-related transcription factor 2 & Mesoderm & 1.92 \\
\hline 20 & SEMA3A & $\begin{array}{l}\text { Sema domain, immunoglobulin domain (lg), } \\
\text { short basic domain, secreted, (semaphorin) 3A }\end{array}$ & Ectoderm & 5.65 \\
\hline 21 & SERPINAI & $\begin{array}{l}\text { "Serpin peptidase inhibitor, clade A } \\
\text { (alpha-I antiproteinase, antitrypsin), member I" }\end{array}$ & Endoderm & 0.59 \\
\hline 22 & SYCP3 & Synaptonemal complex protein 3 & Germ cell marker & 2.93 \\
\hline 23 & SYP & Synaptophysin & Ectoderm & 2.29 \\
\hline
\end{tabular}

Notes: Cutoff of 1.5 -fold up- or downregulation was set. Normalization was carried out using $18 \mathrm{~s}$ rRNA. Expression of each specific gene was calculated using $2^{-(\Delta C T)}$ and was compared with the value obtained for $10 \%$ FBS cultured WJ-MSC.

Abbreviations: bFGF, basic fibroblast growth factor; FBS, fetal bovine serum; HS, human AB serum; WJ-MSC, Wharton's jelly-derived mesenchymal stem cell.

markers in WJ-MSCs compared with BM-MSCs both at early and late passages. ${ }^{28}$ Here, in an effort to compare the stem cell-specific marker profile between the two populations of the WJ-MSCs and to investigate whether there is a shift in the propensity to differentiate towards a particular lineage on switching from FBS to allogeneic HS, we studied the gene expression, with respect to stem cell and early lineage markers, of WJ-MSCs derived from two donors expanded in 5\% $\mathrm{HS}+2 \mathrm{ng} / \mathrm{mL}$ bFGF and $10 \% \mathrm{FBS}$. To our knowledge, there is no previous report comparing the gene expression profile of WJ-MSCs cultured under xeno-free conditions versus the standard condition of $10 \%$ FBS. The fold change in expression of the differentially regulated genes between the two populations varied to some extent between donors. However, the overall expression profile of WJ-MSCs cultured in 5\% $\mathrm{HS}+2 \mathrm{ng} / \mathrm{mL}$ bFGF versus $10 \%$ FBS did not differ much. The increased expression of some ectodermal or endothelial genes by the allogeneic, HS cultured WJ-MSCs, could suggest a stronger propensity to differentiate towards neuronal fate or vascularization compared with the FBS cultured cells. However, further in vitro differentiation or in vitro experiments would be necessary to confirm the same. For a more extensive study, global gene expression analysis needs to be explored. Therefore, MSCs when cultured under different in vitro conditions could possibly adopt a different fate or differentiation potential and thus prove promising for their use in various other clinical indications.

All reported clinical trials to date have employed MSCs expanded in FBS-containing culture medium and they are the best characterized cells. FBS, however, is not a suitable option with regard to patient safety. Again, to be considered appropriate for use in therapeutic applications, utilizing a different serum source should not change the MSCs, with respect to their basic characteristics or gene expression, during the course of the culture. Therefore, in conclusion, pooled, allogeneic human serum can be used successfully to replace FBS for expansion of functional WJ-MSCs. 


\section{Acknowledgments}

This work was fully funded by internal funding of Stempeutics Research Pvt Ltd, Bangalore, India. We are grateful to Dr Praveena Shenoi, Consultant Obstetrician and Gynecologist, Manipal Hospital, for generously providing the umbilical cord samples. We thank Dr Shivaram, Consultant and Chief, Manipal Hospital Transfusion Services, for providing us with the human $\mathrm{AB}$ serum.

\section{Disclosure}

The authors declare no competing financial interests.

\section{References}

1. Caplan AI. Why are MSCs therapeutic? New data: new insight. J Pathol. 2008;217:318-324.

2. Friedenstein AJ, Petrakova KV, Kurolesova AI, Frolova GP. Heterotopic of bone marrow. Analysis of precursor cells for osteogenic and hematopoietic tissues. Transplantation. 1968;6:230-247.

3. Friedenstein AJ, Deriglasova UF, Kulagina NN, et al. Precursors for fibroblasts in different populations of hematopoietic cells as detected by the in vitro colony assay method. Exp Hematol. 1974;2:83-92.

4. Chen MY, Lie PC, Li ZL, Wei X. Endothelial differentiation of Wharton's jelly-derived mesenchymal stem cells in comparison with bone marrowderived mesenchymal stem cells. Exp Hematol. 2009;37(5):629-640.

5. Fong CY, Chak LL, Biswas A, et al. Human Wharton's jelly stem cells have unique transcriptome profiles compared to human embryonic stem cells and other mesenchymal stem cells. Stem Cell Rev. 2011; $7(1): 1-16$

6. Troyer DL, Weiss ML. Wharton's jelly-derived cells are a primitive stromal cell population. Stem Cells. 2008;26(3):591-599.

7. Tonti GA, Mannello F. From bone marrow to therapeutic applications: different behaviour and genetic/epigenetic stability during mesenchymal stem cell expansion in autologous and foetal bovine sera? Int J Dev Biol. 2008;52(8):1023-1032.

8. Selvaggi TA, Walker RE, Fleisher TA. Development of antibodies to fetal calf serum with arthus-like reactions in human immunodeficiency virus-infected patients given syngeneic lymphocyte infusions. Blood. 1997;89(3):776-779.

9. Spees JL, Gregory CA, Singh H, et al. Internalized antigens must be removed to prepare hypoimmunogenic mesenchymal stem cells for cell and gene therapy. Mol Ther. 2004;9(5):747-756.

10. Chachques JC, Herreros J, Trainini J, et al. Autologous human serum for cell culture avoids the implantation of cardioverter-defibrillators in cellular cardiomyoplasty. Int J Cardiol. 2004;95(Suppl 1):S29-S33.

11. Shahdadfar A, Frønsdal K, Haug T, Reinholt FP, Brinchmann JE. In vitro expansion of human mesenchymal stem cells: choice of serum is a determinant of cell proliferation, differentiation, gene expression, and transcriptome stability. Stem Cells. 2005;23(9):1357-1366.

12. Yamaguchi M, Hirayama F, Wakamoto $\mathrm{S}$, et al. Bone marrow stromal cells prepared using $\mathrm{AB}$ serum and $\mathrm{bFGF}$ for hematopoietic stem cells expansion. Transfusion. 2002;42(7):921-927.

Stem Cells and Cloning: Advances and Applications

\section{Publish your work in this journal}

Stem Cells and Cloning: Advances and Applications is an international, peer-reviewed, open access journal. Areas of interest in stem cell research include: Embryonic cell stems; Adult stem cells; Blastocysts; Cordblood stem cells; Stem cell transformation and culture; Therapeutic cloning; Umbilical cord blood and bone marrow cells; Laboratory,
13. Anselme K, Broux O, Noel B, et al. In vitro control of human bone marrow stromal cells for bone tissue engineering. Tissue Eng. 2002;8(6): 941-953.

14. Le Blanc K, Samuelsson H, Lönnies L, Sundin M, Ringdén O. Generation of immunosuppressive mesenchymal stem cells in allogeneic human serum. Transplantation. 2007;84(8):1055-1059.

15. Berney T, Kenyon NS, Alejandro R, Inverardi L, Mintz DH, Ricordi C. Islet transplantation. In: Sperling M, editor. Type I Diabetes: Etiology and Treatment. Totowa, NJ: Humana Press Inc; 2003:529-552.

16. La Rocca G, Anzalone R, Corrao S, et al. Isolation and characterization of Oct-4+/HLA-G+ mesenchymal stem cells from human umbilical cord matrix: differentiation potential and detection of new markers. Histochem Cell Biol. 2009;131(2):267-282.

17. Xu Y, Meng H, Li C, et al. Umbilical cord-derived mesenchymal stem cells isolated by a novel explantation technique can differentiate into functional endothelial cells and promote revascularization. Stem Cells Dev. 2010;19(10):1511-1522.

18. De Bruyn C, Najar M, Raicevic G, et al. A rapid, simple, and reproducible method for the isolation of mesenchymal stromal cells from Wharton's jelly without enzymatic treatment. Stem Cells Dev. Epub 2010 Nov 9.

19. Nekanti U, Mohanty L, Venugopal P, Balasubramanian S, Totey S, Ta M. Optimization and scale-up of Wharton's jelly-derived mesenchymal stem cells for clinical applications. Stem Cell Res. 2010;5(3):244-254.

20. Newman RE, Yoo D, LeRoux MA, Danilkovitch-Miagkova A. Treatment of inflammatory diseases with mesenchymal stem cells. Inflamm Allergy Drug Targets. 2009;8(2):110-123.

21. Kuznetsov SA, Mankani MH, Robey PG. Effect of serum on human bone marrow stromal cells: ex vivo expansion and in vivo bone formation. Transplantation. 2000;70(12):1780-1787.

22. Chase LG, Lakshmipathy U, Solchaga LA, Rao MS, Vemuri MC. A novel serum-free medium for the expansion of human mesenchymal stem cells. Stem Cell Res Ther. 2010;1(1):8.

23. Hartmann I, Hollweck T, Haffner S, et al. Umbilical cord tissue-derived mesenchymal stem cells grow best under GMP-compliant culture conditions and maintain their phenotypic and functional properties. J Immunol Methods. 2010;363(1):80-89.

24. Lindroos B, Boucher S, Chase L, et al. Serum-free, xeno-free culture media maintain the proliferation rate and multipotentiality of adipose stem cells in vitro. Cytotherapy. 2009;11(7):958-972.

25. Bonab MM, Alimoghaddam K, Talebian F, Ghaffari SH, Ghavamzadeh A, Nikbin B. Aging of mesenchymal stem cell in vitro. BMC Cell Biol. 2006;7:14.

26. Sotiropoulou PA, Perez SA, Salagianni M, Baxevanis CN, Papamichail M. Characterization of the optimal culture conditions for clinical scale production of human mesenchymal stem cells. Stem Cells. 2006;24(2): 462-471.

27. Tse WT, Pendleton JD, Beyer WM, Egalka MC, Guinan EC. Suppression of allogeneic T-cell proliferation by human marrow stromal cells: implications in transplantation. Transplantation. 2003;75(3):389-397.

28. Nekanti U, Rao VB, Bahirvani AG, Jan M, Totey S, Ta M. Longterm expansion and pluripotent marker array analysis of Wharton's jelly-derived mesenchymal stem cells. Stem Cells Dev. 2010;19(1): 117-130.

\footnotetext{
Submit your manuscript here: http://www.dovepress.com/stem-cells-and-cloning-advances-and-applications-journal
}

animal and human therapeutic studies; Philosophical and ethical issues related to stem cell research. This journal is indexed on CAS. The manuscript management system is completely online and includes a quick and fair peer-review system. Visit http://www.dovepress.com/ testimonials.php to read real quotes from published authors. 